\title{
Small nucleolar RNA Sf-15 regulates proliferation and apoptosis of Spodoptera frugiperda Sf9 cells
}

Bo Wu ${ }^{\dagger}$, Lei Huang ${ }^{\dagger}$, Wujie Qiu, Xiao Liu, Yawen Shen, Yiping Lu, Zonglin Yang, Xinmei Li, Bin Cui, Shidong Xu, Huili Qiao, Reng Qiu, Lunguang Yao, Yunchao Kan* and Dandan Li* (1)

\begin{abstract}
Background: Small nucleolar RNAs (snoRNAs) function in guiding 2'-O-methylation and pseudouridylation of ribosomal RNAs (rRNAs) and small nuclear RNAs (snRNAs). In recent years, more and more snoRNAs have been found to play novel roles in mRNA regulation, such as pre-mRNA splicing or RNA editing. In our previous study, we found a silkworm C/D box snoRNA Bm-15 can interact with Notch receptor gene in vitro. To further study the function of Bm-15, we cloned its homolog Sf-15 from Spodoptera frugiperda and investigate the function of Sf-15 in Sf9 cells.
\end{abstract}

Results: We showed that knocking down of Sf-15 can inhibit the proliferation, then induce apoptosis of insect S. frugiperda Sf9 cells, but the results were reversed when Sf- 15 was overexpressed. De novo sequencing of transcriptome of Sf9 cells showed that the expression of 21 apoptosis-related genes were increased upon Sf-15 repression. Further

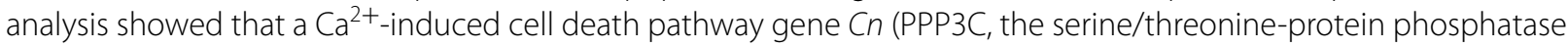
2B catalytic subunit), was significantly increased upon Sf-15 depression but decreased when Sf-15 was overexpressed, which indicated that $\mathrm{Cn}$ might be a potential target of $\mathrm{Sf}-15$.

Conclusions: We conclude that C/D box snoRNA Sf-15 can participate in apoptosis through regulating the expression of $\mathrm{Ca}^{2+}$-induced cell death pathway gene $\mathrm{Cn}$ in Sf9 cells. This is the first time that we found snoRNAs exhibiting dual functions in insect, which reveals a novel layer of ncRNA modulation in cell growth and death.

Keywords: snoRNA, Sf-15, Proliferation, Transcriptome, Sf9 cells

\section{Background}

Small nucleolar RNAs (snoRNAs) are one of the most abundant non-coding RNA species of $60-300$ nucleotides (nt) length and function in guiding 2'-O-methylation (mediated by C/D box snoRNAs) and pseudouridylation (by H/ACA box snoRNAs) in ribosomal RNAs (rRNAs), small nuclear RNAs (snRNAs), tRNAs, and mRNAs $[1,2]$. However, in recent years, a variety of snoRNAs have been found to display novel functions, such as processing molecules into smaller

\footnotetext{
*Correspondence: yckan1974@nynu.edu.cn; lidannytc@hotmail.com ${ }^{\dagger}$ Bo Wu and Lei Huang contributed equally to this work China-UK-NYNU-RRes Joint Laboratory of Insect Biology, Henan Key Laboratory of Insect Biology in Funiu Mountain, Nanyang Normal University, 1638 Wolong Road, Nanyang 473061, Henan, China
}

fragments [3-12] and playing regulatory roles in transcriptional or post-transcriptional levels in human diseases [13-24]. For example, the brain-specific snoRNA, HBII-52, is involved in the regulation of RNA editing and pre-mRNA splicing of serotonin receptor $2 \mathrm{C}$ $\left(5-\mathrm{HT}_{2 \mathrm{C}} \mathrm{R}\right)$ through an $18 \mathrm{nt}$ complementarity to the $5-\mathrm{HT}_{2 \mathrm{C}} \mathrm{R}$ pre-mRNA, and plays important roles in Prader-Willi syndrome [17-19, 25-28]. Furthermore, accumulating evidence suggests that dysregulation of snoRNAs contribute to tumorigenesis [20, 29-32]. For example, accumulation of gas5-generated snoRNAs was related to growth arrest of breast cancer cells [12, 33]. Inhibition of tumor-abundant SNORD78 suppressed the proliferation of non-small cell lung cancer (NSCLC) cells via inducing G0/G1 cell cycle arrest and apoptosis, while SNORD78 overexpression promoted 
cell proliferation [15]. Additionally, SNORD113-1 was found to inactivate the phosphorylation of ERK1/2 and SMAD2/3 in MAPK/ERK and TGF- $\beta$ pathways and suppress tumorigenesis in hepatocellular carcinoma [34], which indicated that the biological function of snoRNAs are various and elusive.

SnoRNAs in lepidopterous insects of silkworm Bombyx mori have been studied by Li et al. [2], but their functional roles have not been fully elucidated. In a previous study, we found a C/D box snoRNA, Bm-15, can interact with a Notch receptor gene in vitro [2]. To further study the function of this snoRNA, we cloned its homolog from Spodoptera frugiperda Sf9 cells, we found that Bm-15 was highly conserved between B. mori and S. frugiperda. Further analysis showed that suppression of Sf-15 inhibited cell growth and promoted cell apoptosis. Transcriptome analysis showed that a serine/threonine-protein phosphatase gene $\mathrm{Cn}$ was a potential target of Sf- 15 . This is the first report that a lepidopterous snoRNA can participate in cell growth through $\mathrm{Ca}^{2+}$-induced cell death pathway, which may provide new clues for understanding the function of snoRNAs.

\section{Results \\ Detect the presence of $\mathrm{Bm}-15$ in $\mathrm{Sf} 9$ cell}

In our previous studies, we found that a $\mathrm{C} / \mathrm{D}$ box snoRNA Bm-15 can interact with a Notch receptor gene in B. mori. To further study the function of Bm-15, we cloned its homolog in S. frugiperda Sf9 cells (whose transfection efficiency is much higher than cell lines of silkworm). We found that Bm-15 was conserved between B. mori and S. frugiperda, with sequence identity of $97 \%$ and similarly secondary structure (Fig. 1a, b). Eukaryotic C/D box snoRNAs guide 2'-O-methylation of rRNAs, snRNAs, and tRNAs through C/D box small nucleolar ribonucleoprotein particles (C/D box snoRNPs), which involves the core proteins of Snu13p/15.5 K, Nop58p/NOP58, Nop56p/NOP56, and Nop1p/Fibrillarin. To determine a

B. mori

S. frugiperda

Consensus

B. mori

S. frugiperda

Consensus

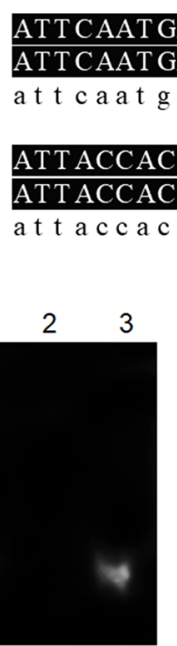

d

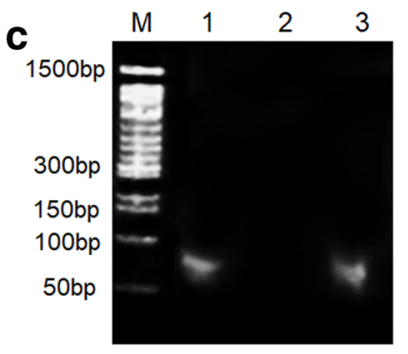

f

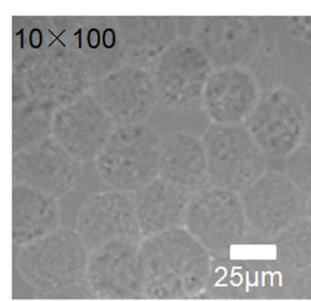

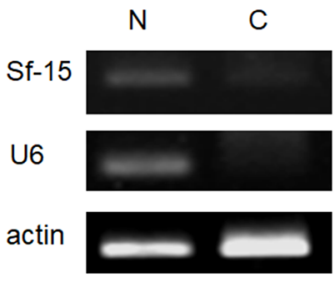

DAPI

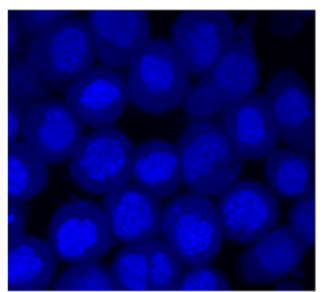

U3

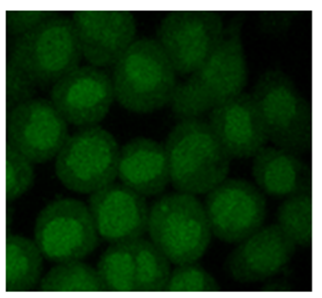

b

40
40

79

79
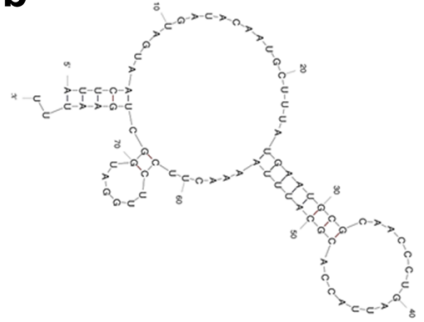

e

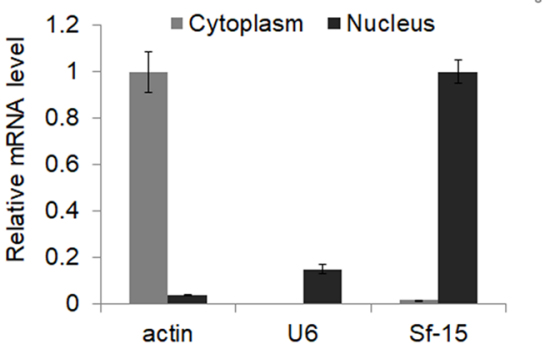

Sf-15

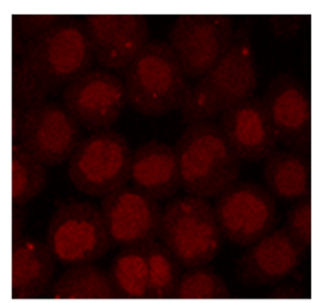

Merge

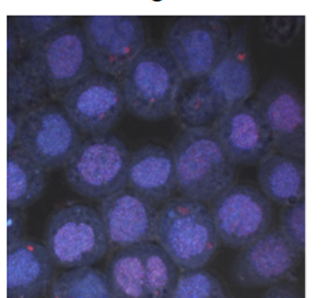

Fig. 1 Characterization of Sf-15 in Sf9 cells. a Sequence identity of Bm-15 between B. mori and S. frigiperda. b Secondary structure of Sf-15 in S. frigiperda. c Abundance of Sf-15 that was pulled down by the antibody of Fibrillarin. M, 100 bp DNA ladders. 1-3 represented PCR products that were amplified from RNAs being pulled down by anti-Fibrillarin, serum and input, respectively. $\mathbf{d}$ Expression of Sf-15 in nucleus and cytoplasm of Sf9 cells. N Nucleus, C cytoplasm. e Relative expression of Sf-15 in nucleus and cytoplasm by quantitative real-time PCR. Results were calculated by relative Ct of different genes. $\mathbf{f}$ Fluorescent in situ hybridization of Sf-15. Phase represented cells observed under white light. U3 snoRNA was used as a nucleolar marker and was visualized by employing a $5^{\prime}$-FITC-labeled antisense oligonucleotide. The in situ hybridization probes of Sf-15 were labeled with Cy3 at the $5^{\prime}$ end, the nucleus was stained with DAPI. Scale bar represented $25 \mu \mathrm{m}$ 
the characteristics of Sf-15, we used the antibody against Fibrillarin, one of the most important core protein of C/D box snoRNPs to pull down RNAs. Results showed that $\mathrm{Sf}-15$ can be fished out by Fibrillarin (Fig. 1c), which suggested that Sf-15 might be a real snoRNA.

Next, the cellular location of Sf-15 was detected in Sf9 cells. Results showed that Sf-15 was highly existed in the nucleus of Sf9 cells (Fig. 1d, e). Moreover, Immunofluorescence in situ hybridization (FISH) of Sf-15 with Cy3-labeled antisense probes confirmed that $\mathrm{Sf}-15$ was dominantly in nucleus of Sf9 cells (Fig. 1f).

\section{Repression of Sf-15 blocks proliferation and promotes apoptosis and death of Sf 9 cells}

snoRNAs are located in the nucleoli, the traditional methods of RNA interference (RNAi) such as dsRNAs or siRNAs can hardly work, but RNase H1-dependent antisense oligonucleotides (ASOs) can be easily transported into the nucleus [35-38]. So here the modified ASO of Sf- 15 was used to repress the expression of Sf-15 in Sf9 cells. Results showed that the expression of Sf-15 was inhibited by nearly $33 \%$ after $24 \mathrm{~h}$ transfection, and reached to $75 \%$ after $72 \mathrm{~h}$ transfection of ASO (Fig. 2a), which indicated that the modified ASO can effectively knock down the expression of Sf-15.

Cell morphology observations with DAPI showed that Sf9 cells with chromatin condensation and apoptotic bodies increased by approximately $9 \%$ after $72 \mathrm{~h}$ transfection of Sf-15 antisense oligonucleotides (Fig. 2b, c, number of examined cells of each sample is 1500). Moreover, flow cytometry assay revealed that the apoptotic rate of Sf9 cells increased by approximately $3.2 \%$, and the death rate of cells increased $11.1 \%$ after $72 \mathrm{~h}$ transfection of Sf-15 ASO (Fig. 2d).

To further study the regulator roles of Sf-15 in cell growth, the overexpression vector $p B a c[A 3-E G F P-A 3-$ $S f-15]$ was used. Results showed that the expression of Sf-15 increased 5 times after $48 \mathrm{~h}$ transfection, while to 10 times after $72 \mathrm{~h}$ transfection (Fig. 2e), which indicated that the Sf-15 was successfully overexpressed. Then the cell proliferation rate was detected by WST- 8 (the analogue of MTT), results showed that the cell growth rate was impaired by nearly $19 \%$ after $48 \mathrm{~h}$ transfection of Sf-15 ASO, but increased 38\% as ectopic overexpression of Sf-15 at the same time-point (Fig. 2f), which indicated that $\mathrm{Sf}-15$ might participate in the regulation of cell proliferation.

\section{Transcriptome analysis of knocking down and ectopic expression of Sf-15 in Sf9 cells}

To determine the roles that Sf- 15 plays in cell growth, RNA libraries of normal Sf9 cells (named Sf9), cells with repression (named ASO) and overexpression of Sf-15 (named Sf-15) were constructed respectively. De novo transcriptome sequencing was used to detect the differential expression of protein coding genes. A total of $59,297,730,59,614,986$ and 57,045,606 raw reads were obtained from the three libraries of Sf9, ASO and Sf-15, respectively. After removing the low-quality reads and adaptor sequences, a total of $77,578,82,715$, and 73,398 contigs were obtained from the three libraries, respectively (Table 1). Finally, we got 46,529, 47,231 and 43,032 unigenes from Sf9, ASO and Sf-15, respectively, with an average length of $750 \mathrm{bp}$.

A total of 9291 (34.81\%) unigenes were classified into 3 main GO categories (biological process, cellular component, and molecular function) and 57 subcategories (Fig. 3a, Additional file 1: Table S1). The large part of unigenes were assigned to cellular process, with a percentage of $14.98 \%$, then followed by single-organism process (11.51\%) and metabolic process (11.44\%). A total of 17,700 COG functional annotations were obtained and classified into 25 functional categories (Fig. 3b).

Expression analysis showed that 355 unigenes were significantly upregulated and 1099 were significantly downregulated when Sf-15 was overexpressed. While 1372 were upregulated and 1051 were downregulated upon Sf-15 repression (Fig. 4b, Additional file 1: Table S1). Further analysis showed that the expression of 1111 unigenes had reverse expression pattern under Sf- 15 overexpression and repression, the expressin of 909 genes were significantly increased when Sf-15 was knocked down, while 202 unigenes were significantly decreased when Bm-15 was repressed (Additional file 1: Table S1 and Additional file 2: Fig. S1). Interestingly, 21 genes of the apoptosis pathway were upregulated upon Sf-15 repression (Fig. 4a and Additional file 1: Table S1), but with reverse expression pattern when Sf-15 was overexpressed, which indicated Sf-15 might participate in the apoptosis pathway.

RT-PCR was carried out to further verify the sequencing results. We found that the expression of $p 53$ and caspase-1 were increased when Sf-15 was knocked down, but decreased when Sf-15 was ectopic expressed in Sf9 cells. While the apoptosis inhibitor (IAP) gene had a contrary expression pattern (whose expression was decreased upon $\mathrm{Sf}-15$ repression and increased when Sf-15 was overexpressed) (Fig. 4d), which indicated that $\mathrm{Sf}-15$ might play roles in the process of cell growth and apoptosis.

\section{Sf-15 accelerate cell proliferation by targeting to $\mathrm{Cn}$ gene}

To further study the targets of Sf-15, KEGG pathway of differentially expressed genes were analyzed, we found that 2 of the 21 apoptosis-related genes were significantly increased when $\mathrm{Bm}-15$ was knocked down, especially the $C n$ gene (PPP3C, the catalytic subunit of $P P 2 B$, which 

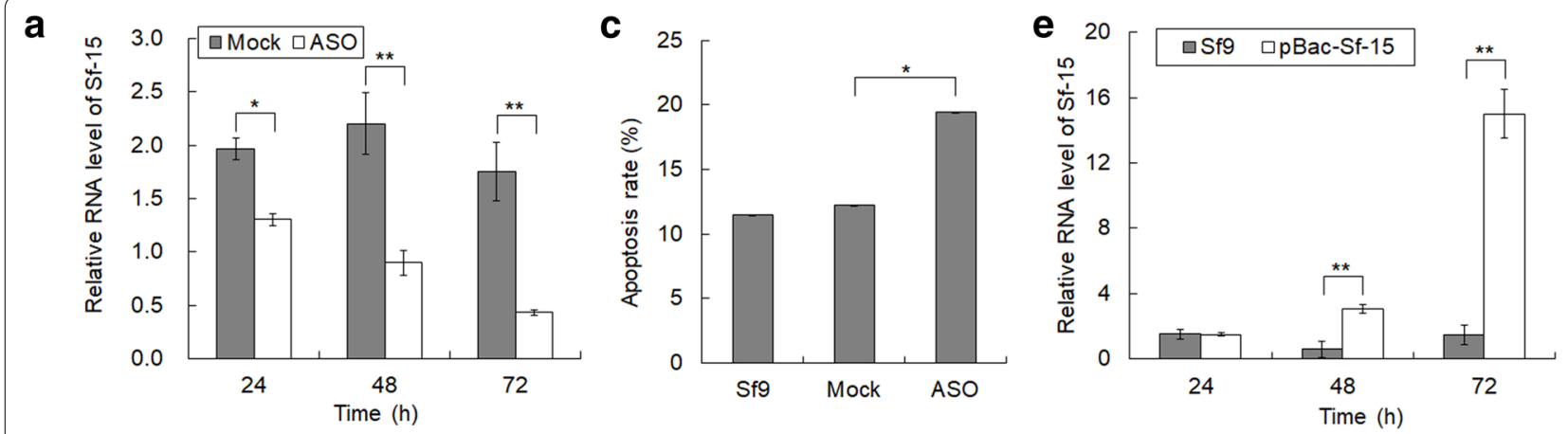

b
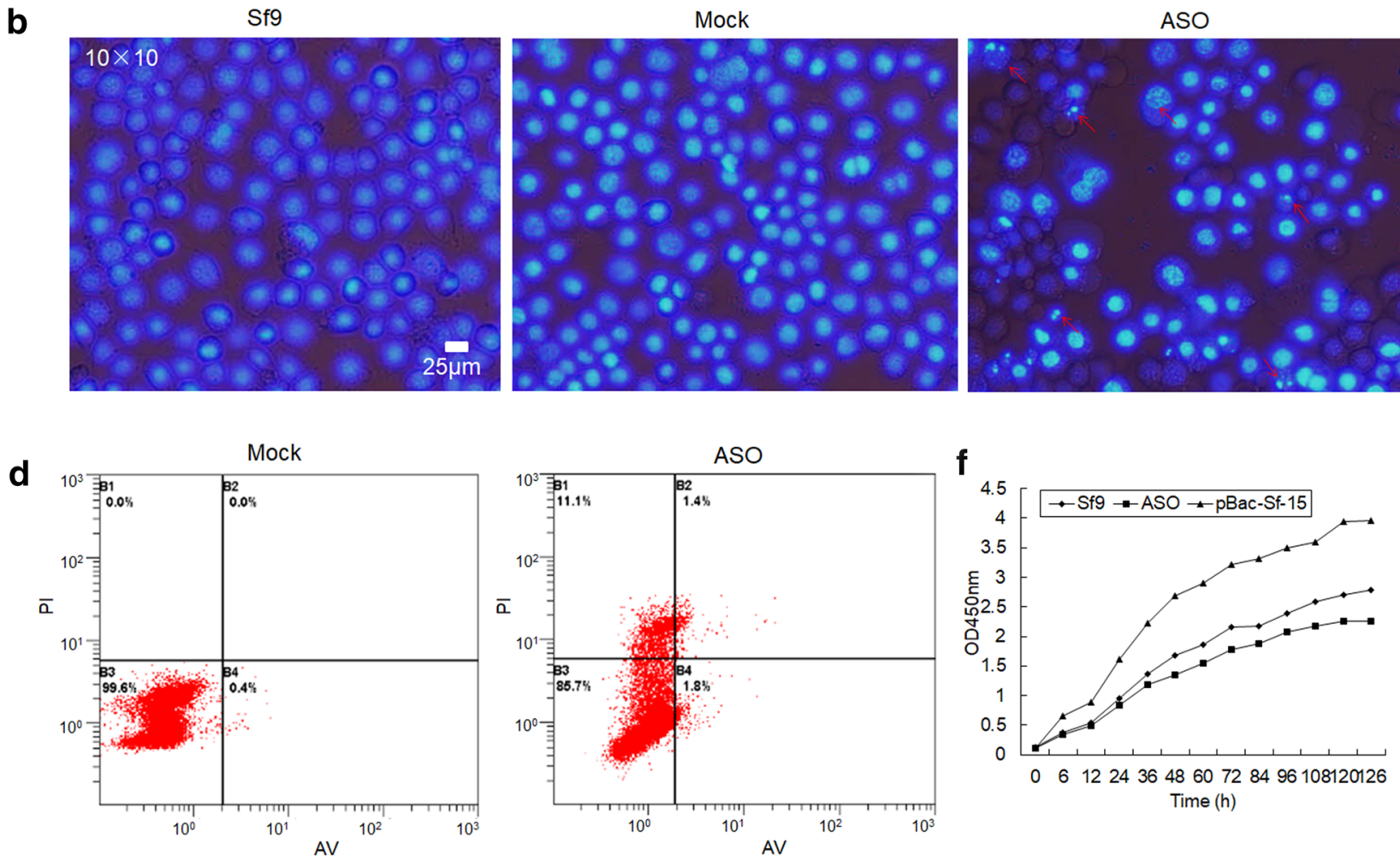

Fig. 2 Knocking down of Sf-15 inhibited the proliferation and induced apoptosis and death of Sf9 cells. a Relative expression of Sf-15 after ASO transfection at different time-points. Mock means Sf9 cells transfected with antisense oligonucleotide of negative control (NC), ASO menas cells transfected with antisense oligonucleotides of Sf-15. U6 was used as an internal control. b DAPI staining showed chromatin condensation and apoptotic bodies in Sf9 cells after $72 \mathrm{~h}$ transfection of Sf-15 antisense oligonucleotide. Sf9 represented normal Sf9 cells, Mock and ASO refers to cells transfected with ASO of NC and Sf-15, respectively. Red arrows showed the apoptotic bodies. Scale bar represented $25 \mu \mathrm{m}$. c Apoptosis rate of cells that were transfected with antisense oligonucleotide of NC (Mock) and Sf-15 (ASO), respectively. $\mathbf{d}$ Apoptotic rates determined by Annexin-V/PI stain after transfection with antisense oligonucleotide of NC (Mock) and Sf-15 (ASO), respectively. The B3, B2, B4 and B1 quadrants in each panel represent the populations of normal, early and late apoptotic, and apoptotic necrotic cells, respectively. e Relative expression of Sf-15 after transfection of pBac[A3-EGFP-A3-Sf-15] (pBac-Sf-15) vector at different time-points. U6 was used as an internal control. f Growth curves of Sf9 cells detected by WST-8 method upon Sf-15 overexpression and repression. 6, 12, 24, 36, 48, 60, 72, 84, 96, 108, 120 and $126 \mathrm{~h}$ represented different timepoint after transfection of Sf-15 antisense oligonucleotides (ASO) and pBac[A3-EGFP-A3-Sf-15] (pBac-Sf-15) respectively. Sf9 means normal Sf9 cells

encode a calcium-dependent serine/threonine phosphatase), with the $\log 2$ ratio of 7.5 (Fig. 4c). Quantitative real-time PCR results of $\mathrm{Cn}$ gene confirmed the results of transcriptome sequencing (Fig. 4d), which indicated that $\mathrm{Cn}$ might be a functional target of $\mathrm{Sf}-15$. In the $\mathrm{Ca}^{2+}$ induced cell death pathway, $\mathrm{Bad}(\mathrm{Bcl}-2 / \mathrm{Bcl}-\mathrm{xL}$-associated 
Table 1 Summary of De novo transcriptome sequencing

\begin{tabular}{|c|c|c|c|c|c|c|c|c|c|}
\hline Samples & $\begin{array}{l}\text { Total raw } \\
\text { reads }\end{array}$ & $\begin{array}{l}\text { Total clean } \\
\text { reads }\end{array}$ & $\begin{array}{l}\text { Total clean } \\
\text { nucleotides } \\
\text { (nt) }\end{array}$ & $\begin{array}{l}\text { Contig } \\
\text { number }\end{array}$ & $\begin{array}{l}\text { Contig } \\
\text { length (nt) }\end{array}$ & $\begin{array}{l}\text { Mean } \\
\text { contig } \\
\text { length (nt) }\end{array}$ & $\begin{array}{l}\text { Total } \\
\text { unigene } \\
\text { number }\end{array}$ & $\begin{array}{l}\text { Total } \\
\text { unigene } \\
\text { length (nt) }\end{array}$ & $\begin{array}{l}\text { Mean } \\
\text { unigene } \\
\text { length } \\
\text { (nt) }\end{array}$ \\
\hline Sf9 & $59,297,730$ & $55,266,566$ & $4,973,990,940$ & 77,578 & $30,360,233$ & 391 & 46,529 & $35,208,893$ & 757 \\
\hline ASO & $59,614,986$ & $55,185,956$ & $4,966,736,040$ & 82,715 & $31,014,680$ & 375 & 47,231 & $36,395,105$ & 771 \\
\hline Sf-15 & $57,045,606$ & $52,924,702$ & $4,763,223,180$ & 73,398 & $27,797,369$ & 379 & 43,032 & $31,685,987$ & 736 \\
\hline
\end{tabular}

death promoter) was the downstream gene of $\mathrm{Cn}$, the protein of which can be dephosphorylated by $\mathrm{Cn}$. So the change of $\mathrm{Bad}$ was also detected by real-time PCR, results showed that the expression of $\mathrm{Bad}$ was decreased when Sf-15 was overexpressed, but with little variation upon Sf-15 repression, which was consistent with the transcriptome sequencing result, which indicated that $\mathrm{Cn}$ might regulate its downstream gene $\mathrm{Bad}$ not only in mRNA level but in the level of protein dephosphorylation. Further analysis showed that there is no sequence complement between $\mathrm{Sf}-15$ and $\mathrm{Cn}$, then how they interact with each other need much more evidence.

\section{Discussion}

Regulation of apoptosis is essential for many developmental processes (Testa 2004). Recent years, besides protein coding genes, the non-coding RNAs were also found to be participated in cell growth and apoptosis in different organisms. For example, Gas5 (growth arrest specific 5), a tumor-suppressive long noncoding RNA, and a small nucleolar RNA (snoRNA) host gene similar to UHG (U22 host gene), encoded 10 human box C/D snoRNAs, can potently upregulates the transcription of tumor necrosis factor (TNF)-related apoptosis inducing ligand (TRAIL) by inducing H3K4 methylation/H3K27 demethylation. Overexpression of Gas5 transcripts were shown to induce growth arrest and apoptosis in several mammalian cell lines [12, 33, 39, 40]. Moreover, in Drosophila, the GAS5-origined scaRNA Dm46E3 had reverse expression pattern to its antisense protein coding gene eiger (who plays a key role in cell differentiation, apoptosis and immune response), might participate in embryogenesis of Drosophila through target to U1b snRNA, which indicated the novel role of snoRNAs in cell growth [41].

In our study, knocking down the C/D box snoRNA Sf-15 inhibited the proliferation and induced apoptosis and death of $\mathrm{Sf} 9$ cells. Then the expression of a serine/threonine-protein phosphatase 2B $(P P 2 B)$ catalytic subunit gene $C n$, was significantly increased upon $\mathrm{Sf}-15$ depression. $\mathrm{Cn}$ is a $\mathrm{Ca}^{2+}$-dependent protein phosphatase (also known as calcineurin), which can catalyze the dephosphorylation of its downstream gene Bcl-2/
Bcl-xL-associated death promoter $(\mathrm{Bad})$, the dephosphorylated Bad can interact with Bcl-XL to initiate cell apoptosis and death [42]. The accumulation of $\mathrm{Cn}$ upon Sf-15 repression might increase the dephosphorylation level of Bad and initiate apoptosis and death of Sf9 cells. To investigate the mechanism that how $\mathrm{Sf}-15$ interact with $\mathrm{Cn}$, the sequences of Sf- 15 and $C n$ were aligned in both strands, the secondary structure of them were also compared. But there was low potential of reverse complement between Sf-15 and $\mathrm{Cn}$ (data not shown). Moreover, we also analyzed whether Sf- 15 had the potential to be precursor of miRNAs, but the answer was no. So how Sf-15 interact with $C n$, and how Sf-15 participate in apoptosis through $\mathrm{Ca}^{2+}$-induced apoptosis pathway still need much more evidence.

Therefore, we hypothesize that Sf-15 might participate in the $\mathrm{Ca}^{2+}$ induced apoptosis pathway through regulating the expression of $\mathrm{Cn}$ and its downstream genes. Exploring how this snoRNA-mediated activity in apoptosis will be of great interest. Our finding of the apoptotosis-related snoRNA reveals a novel layer of snoRNA modulation in cell death program.

\section{Conclusions}

More and more evidence showed that besides guiding 2'-O-methylation and pseudouridylation of rRNAs, snoRNAs can also interact with mRNAs, such as to be precursor of miRNAs, or compete with splicing factors at the splicing sites of pre-mRNAs. Here we found that knocking down of C/D box snoRNA Sf-15 induced apoptosis and death of Sf9 cells, further analysis showed that a $\mathrm{Ca}^{2+}$-induced cell death pathway gene $\mathrm{Cn}$ might be a potential target of $\mathrm{Sf}-15$, which provide new clues for the functional excavation of snoRNAs in the future.

\section{Methods}

Cell culture and transfection

Sf9 cells were maintained in Grace medium (Thermo Fisher Scientific) containing $10 \%$ fetal bovine serum (FBS) (Thermo Fisher Scientific) at $28^{\circ} \mathrm{C}$. Cells were seed 


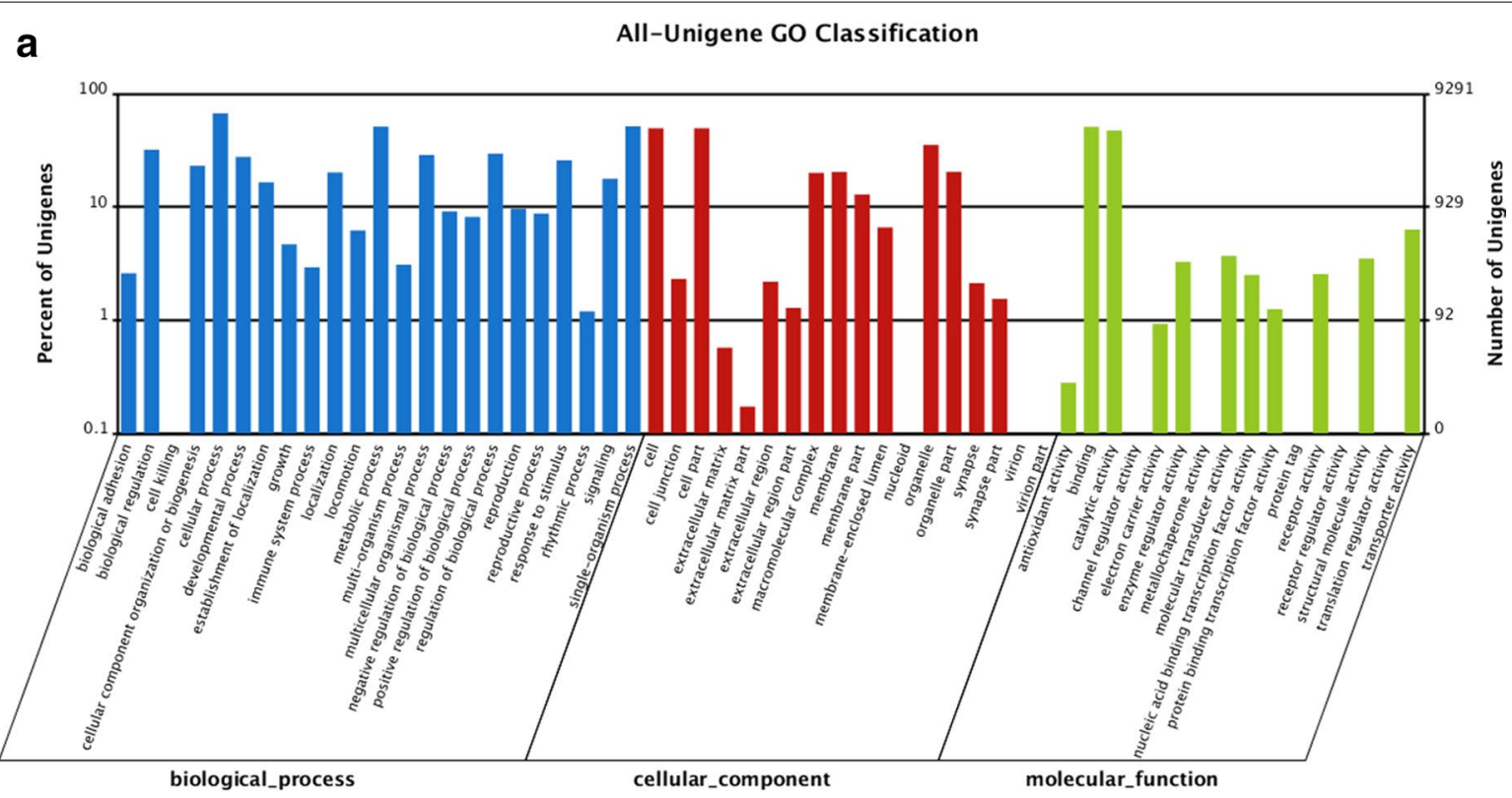

b COG Function Classification of All-Unigene.fa Sequence

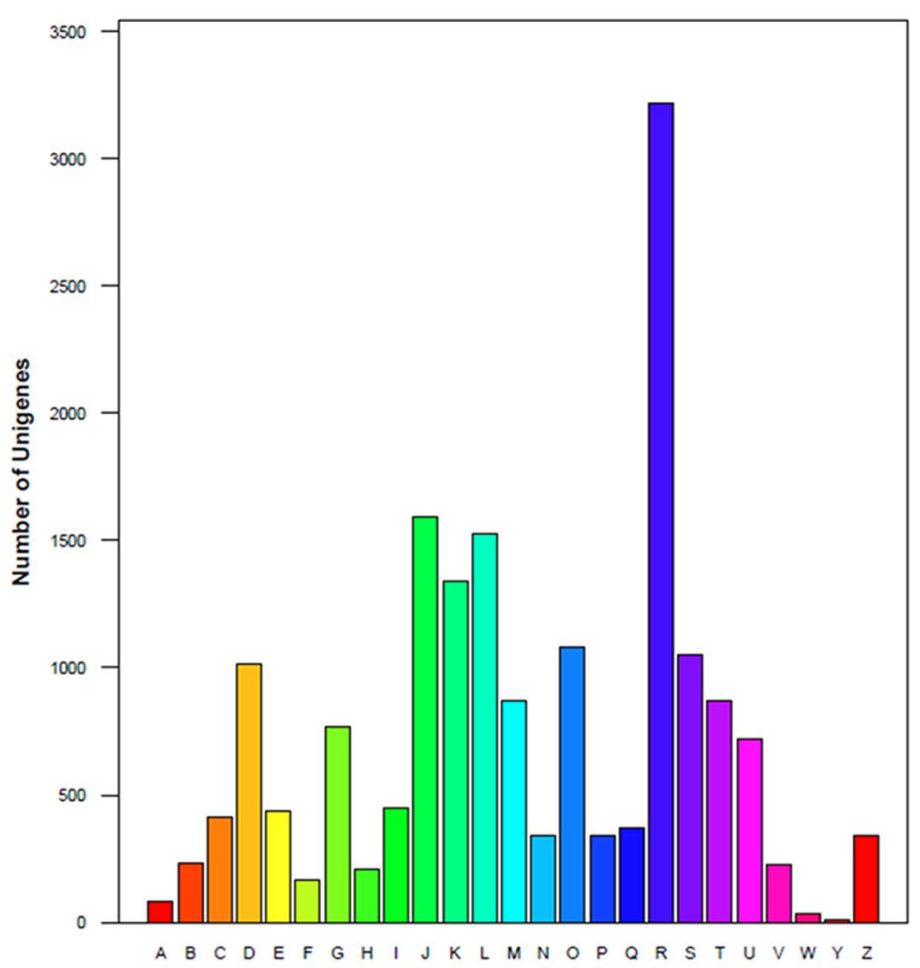

Function Class

A: RNA processing and modification

B: Chromatin structure and dynamics

C: Energy production and conversion

D: Cell cycle control, cell division, chromosome partitioning

E: Amino acid transport and metabolism

F: Nucleotide transport and metabolism

G: Carbohydrate transport and metabolism

$\mathrm{H}$ : Coenzyme transport and metabolism

I: Lipid transport and metabolism

$\mathrm{J}$ : Translation, ribosomal structure and biogenesis

K: Transcription

L: Replication, recombination and repair

M: Cell wall/membrane/envelope biogenesis

$\mathrm{N}$ : Cell motility

O: Posttranslational modification, protein turnover, chaperones

$\mathrm{P}$ : Inorganic ion transport and metabolism

$\mathrm{Q}$ : Secondary metabolites biosynthesis, transport and catabolism

$\mathrm{R}$ : General function prediction only

$S$ : Function unknown

T: Signal transduction mechanisms

U: Intracellular trafficking, secretion, and vesicular transport

$\mathrm{V}$ : Defense mechanisms

W: Extracellular structures

Y: Nuclear structure

Z: Cytoskeleton

Fig. 3 Functional classification of all unigenes from transcriptome sequencing results. a GO analysis of all unigenes in Sf9 cells. b COG function classification of all unigenes in Sf9 cells 


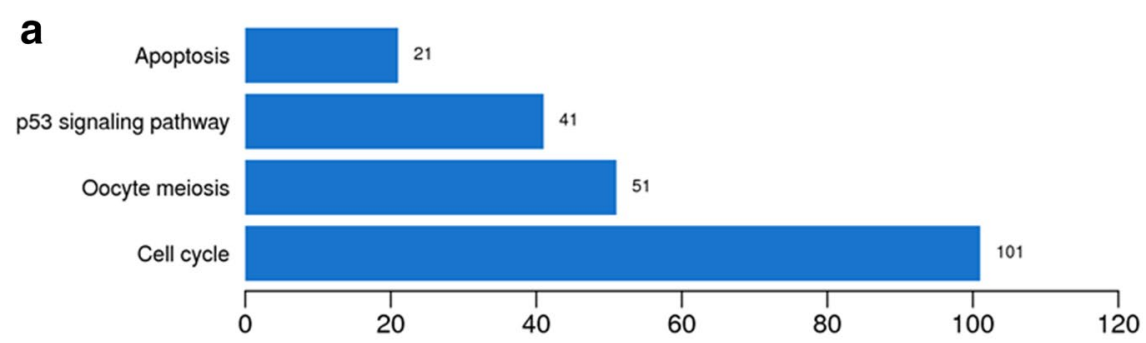

Number of unigenes

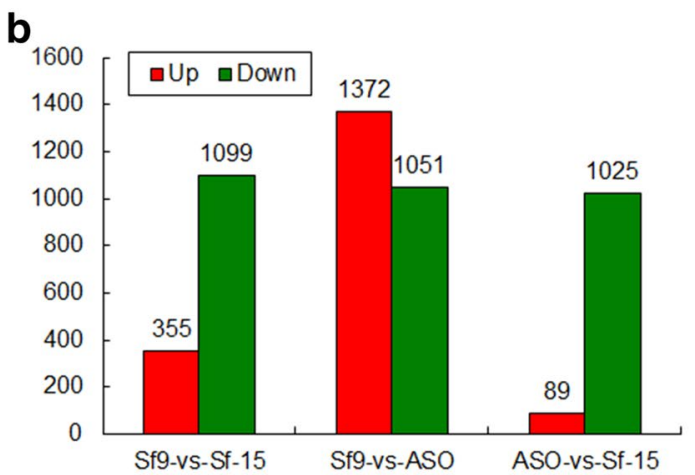

C

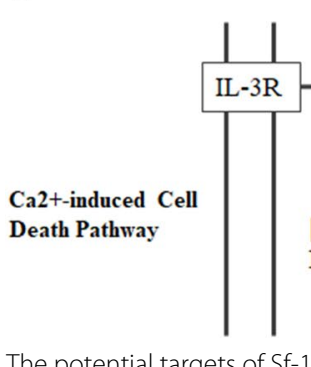

Fig. 4 The potential targets of Sf-15. a KEGG pathway analysis of genes related to cell growth and death, which showed the numbers of genes in different signal pathways. $\mathbf{b}$ Numbers of genes with significantly varied expression upon Sf-15 repression and overexpression in Sf9 cells. Sf9 means normal Sf9 cells, Sf-15 represents cells transfected with the overexpression vector of pBac[A3-EGFP-A3-BmSf-15] (pBac-Sf-15). ASO means cells transfected with the antisense oligonucleotide of Sf-15. Red means the expression of genes were increased, green means the expression of genes were decreased. $\mathbf{c}$ Detailed information of genes in $\mathrm{Ca}^{2+}$ induced cell death pathway after overexpression of Sf-15 in Sf9 cells. Green box represented the expression of this gene was decreased upon Sf-15 overexpression but increased when Sf-15 was knocked down. $\mathbf{d}$ Quantitative real-time PCR results of genes in apoptosis pathway after transfection of Sf-15 antisense oligonucleotides (ASO) and overexpression vector $p B a c[A$ 3-EGFP-A3-Sf-15] (pBac-Sf-15). IAP represented the inhibitor of apoptosis gene. actinA3 was used as an internal control

in 24-well plates to 70-80\% confluency and further transfected with $2 \mu \mathrm{g}$ of plasmid DNA or $50 \mathrm{nmol} / / \mathrm{L}$ antisense oligonucleotides (ASO) employing Lipofectamine LTX and Plus Reagents (Thermo Fisher Scientific).

\section{RNA immunoprecipitation (RIP)}

RNA immunoprecipitation (RIP) was followed the protocol of Selth et al. [43] with some modifications. Approximately $10^{7}$ Sf9 cells were cross-linked with $1 \%$ formaldehyde, the immunoprecipitation of Fibrillarincontaining protein complex was prepared with Protein

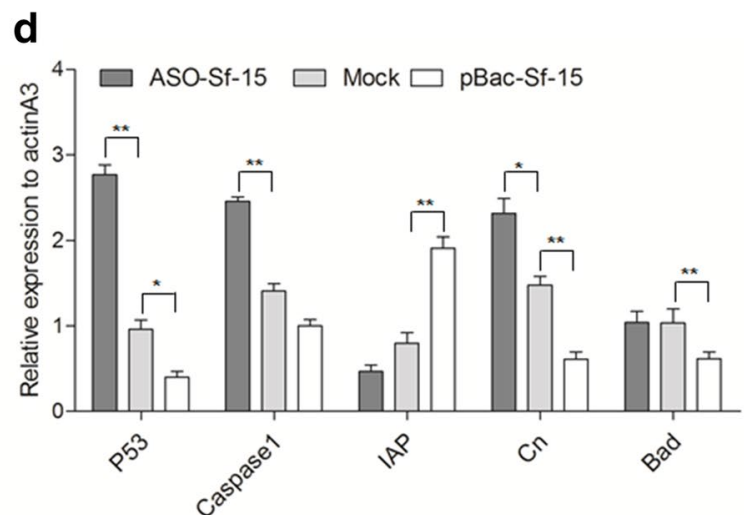

Homodimer
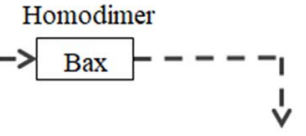

ptosis

$\hat{\text { I }}$

1 
cell lysate before immunoprecipitation (named as input) was used as the positive control.

\section{Nuclear and cytoplasmic RNA isolation and RT-PCR}

The nuclear and cytoplasmic RNAs of Sf9 cells were extracted by Cytoplasmic \& Nuclear RNA Purification Kit (Norgene), cDNAs were generated with 50 ng random hexamer primers (for ncRNAs) or oligo $\mathrm{d}(\mathrm{T})_{15}$ primers (for protein-coding genes) using the Reverse Transcription System (Promega). PCR was performed as following, $94{ }^{\circ} \mathrm{C}$ for $5 \mathrm{~min}$, then at $94{ }^{\circ} \mathrm{C}$ for $30 \mathrm{~s}, 55^{\circ} \mathrm{C}$ for $30 \mathrm{~s}$, and $72{ }^{\circ} \mathrm{C}$ for $30 \mathrm{~s}$ with 25 cycles. The primers used for Sf- 15 , U6 and actinA3 are listed in Additional file 2: Table S2. $\mathrm{U} 6$ and actinA3 were used as nuclear and cytoplasmic control, respectively. PCR products were detected by $2 \%$ agarose gel.

\section{Fluorescent in situ hybridization (FISH)}

FISH of Sf-15 in Sf9 cells was performed according to the method of Querido et al. [44]. Probe of Sf-15 used for in situ hybridization was as the following, CATCCAAGG AAGTTTGAAATGCGTGGTAATCATGGTTGCGCA TTCATAAAGCATTGTA with Cy3-labeled at the $5^{\prime}$ end (TaKaRa). U3 was used as an internal control with FITClabeled at the $5^{\prime}$ end (TaKaRa), nucleus was stained with DAPI. Localization of the Sf- 15 was analyzed by fluorescent microscopy using laser scanning confocal microscope TE2000-E (Nikon).

\section{Plasmids construction and probe synthesis}

Sf-15 was sub-cloned into the $p B a c[A 3-E G F P]$ to construct the overexpression vector. Firstly, the actin $A 3$ promoter was cloned from the plasmid of $p B a c[A 3-E G F P]$ with endonuclease BamHI and NocI, the fragment of Sf-15 was cloned from cDNA of Sf9 with NocI and KpnI at the $5^{\prime}$ and $3^{\prime}$ ends. actinA3 promoter and Sf-15 were sub-cloned into the $P F B D M$ vector, which constructed the vector of $p F B D M[A 3-S f-15]$. Next, $p F B D M$ [A3-Sf-15] was digested by BamHI and BglII and ligated to $p B a c[A 3-$ EGFP], which constructed the $p B a c[A 3-E G F P$-A3-Sf-15] vector. These constructs were identified by sequencing performed by a commercial service provider (AuGCT Biotech).

To knock down the expression of Sf-15, the antisense oligonucleotides (ASO) of Sf-15 were used. ASO are designed in a 5-10-5 gapmer configuration (a chimeric antisense oligonucleotide that contains a central block of deoxynucleotide monomers sufficiently long to induce $\mathrm{RNase} \mathrm{H}$ cleavage, gapmer is flanked by blocks of 2'-O-methoxyethol (MOE) modified ribonucleotides or other artificially modified ribonucleotide monomers can protect the internal block from nuclease degradation [45]), with the sequence of ugguuGCGCATTCATaaagc, the first and last five nucleotides were ribonucleic acids with MOE modification, the middle ten nucleotides were deoxyribonucleic acids, and the total probe had phosphorothioate (PS) chemical modification with Cy5-labeled at the $5^{\prime}$ end.

\section{Cell proliferation and apoptosis assays}

Cell proliferation rate was detected using the Enhanced Cell Counting Kit-8 (Beyotime). Sf9 cells were transfected with $p B a c[A 3-E G F P-A 3-S f-15]$ vector and ASO to overexpress and knock down of Sf-15, cells were collected at 6, 12, 24, 36, 48, 60, 72, 84, 96, 108, 120 and $126 \mathrm{~h}$ after transfection, 2000 cells were seeded in a 96-well plate in $100 \mu \mathrm{l}$ of culture medium and incubated with $10 \mu \mathrm{l}$ WST-8 (2-(2-methoxy-4-nitrophenyl)-3-(4nitrophenyl)-5-(2,4-disulfophenyl)-2H-tetrazolium, monosodium salt) mixture, the analogue of MTT (3-(4,5-Dimethylthiazol-2-yl)-2,5-diphenyltetrazolium bromide), for $2 \mathrm{~h}$. The absorbance of each sample was measured using a microplate reader at a wavelength of $450 \mathrm{~nm}$. Each timepoint had three samples, each sample had three repeats.

Cell apoptosis detected by DAPI staining was carried out as following, Sf9 cells were collected after $72 \mathrm{~h}$ transfection of Sf-15 ASO, the nucleus was stained with DAPI, the chromatin condensation and apoptotic bodies were captured by fluorescent microscopy TE2000-E (Nikon).

The apoptosis rates of Sf9 cells were detected with the Annexin V-FITC cell apoptosis detection kit (Beyotime). $1 \times 10^{5}$ cells were collected and stained with Annexin V-FITC and Propidium Iodide (PI), cells were incubated at room temperature for $20 \mathrm{~min}$ in dark place, then the apoptosis rates were detected on FC-500 flow cytometer (Beckman Coulter).

\section{Quantitative real-time PCR}

Total RNA was extracted from different samples of Sf9 cells with TRIzol (Thermo Fisher Scientific) method. Synthesis of cDNA was performed with $2 \mu \mathrm{g}$ of total RNA and $50 \mathrm{ng}$ random hexamer primers (for ncRNAs) or oligo $\mathrm{d}(\mathrm{T})_{15}$ primers (for protein-coding genes) using the Reverse Transcription System (Promega). The primers used for ncRNAs and protein coding genes are listed in Additional file 2: Table S2. Quantitative realtime PCR was carried out using the FS Universal SYBR Green Master (Roche) on the CFX96 ${ }^{\mathrm{TM}}$ Real-Time PCR Detection System (Bio-Rad). The thermal cycling consists a denaturation step at $95{ }^{\circ} \mathrm{C}$ for $10 \mathrm{~min}$, then 40 
cycles at $95{ }^{\circ} \mathrm{C}$ for $15 \mathrm{~s}, 55 / 58{ }^{\circ} \mathrm{C}$ for $30 \mathrm{~s}$, and $72{ }^{\circ} \mathrm{C}$ for $30 \mathrm{~s}$. Single PCR product was confirmed with the heat dissociation protocol at the end of the PCR cycles. The target gene abundance in each sample was normalized based on U6 (for ncRNAs) or actinA3 (for proteincoding genes) levels using the formula $\Delta \mathrm{Ct}=\mathrm{Ct}$ target gene-CtU6 or actinA3. All experiments were conducted in three independent triplicates, each sample had three repeats.

\section{Illumina sequencing and de novo assembly}

Total RNAs were extracted from normal Sf9 cell as well as cells being transfected with $\mathrm{Sf}-15 \mathrm{ASO}$ and $p B a c[A 3-$ EGFP-A3-Sf-15] with TRIzol method. Transcriptome sequencing was performed using Illumina $\mathrm{HiSeq}^{\mathrm{TM}}$ 2000. Raw reads with $3^{\prime}$-adaptors and repeating reads were removed, while nucleotides with a quality score lower than 20 were trimmed from the end of raw reads. Then, de novo assembly of the clean reads was conducted with Trinity (release-20130225), TGICLL (version 2.1) and Phrap (Release 23.0) programs to generate non-redundant unigenes.

\section{Bioinformatic analysis}

Clean reads were aligned to NCBI non-redundant (NR) protein database, Swiss-Prot, KEGG, and Cluster of Orthologous Group (COG) databases using Blastx $(E$-value $\leq 1 \mathrm{E}-05)$. Unigene sequences were aligned to protein databases (NR, Swiss-Prot, KEGG and COG) to retrieve proteins with the highest sequence similarity to the given unigenes as their protein functional annotations. Furthermore, Blast2GO (version 2.5.0) [46] was used to obtain Gene Ontology (GO) annotation of unigenes with NR database. The WEGO software [47] was then used to perform functional classification of GO term for all unigenes and to comprehend the distribution of gene functions. The unigene sequences were aligned to the COG database to predict and classify possible functions. Pathway assignments were performed according to the KEGG database. The calculation of unigene expression was based on the FPKM method. We named genes with significantly differential expression when the $\log 2$ ratio of FPKM is more than 1. Genome sequence of $B$. mori and S. frugiperda were downloaded from SilkDB [48] and SPODOBASE $[49,50]$.

Sequence identity of Bm-15 between B. mori and $S$. frugiperda were aligned with the software of ClusterW (version 1.83). The secondary structure of $\mathrm{Sf}-15$ was predicated by Mfold (Version 3.6) [51].

\section{Additional files}

Additional file 1: Table S1. Differentially expressed gene list upon Sf-15 overexpression and repression. ASO and Sf-15 means cells were transfected with antisense oligonucleotides of Sf-15 and overexpression vector pBac[A3-EGFP-A3-Sf-15], respectively.

Additional file 2: Fig. S1. Functional classification of genes with reverse expression pattern upon Bm-15 repression and overexpression. (A) GO classification of genes with expression being increased as Sf-15 was knocked down but decreased when Sf-15 was overexpressed. (B) GO classification of genes with expression being decreased when $\mathrm{Sf}-15$ was knocked down but increased when Sf-15 was overexpressed. (C) Statistic analysis of the numbers of genes in figure A. (D) Statistic analysis of the numbers of genes in figure B. ASO and Sf-15 means cells were transfected with antisense oligonucleotides of $\mathrm{Sf}-15$ and overexpression vector pBac[A3-EGFP-A3-Sf-15], respectively. Up means the expression of genes were increased, down means the expression of genes were decreased. Table S2. Primer set used in the experiment. Table S3. Unigenes were subjected to Blastx against public protein database in Sf9 cells.

\section{Abbreviations}

ncRNA: non-coding RNA; snoRNA: small nucleolar RNAs; rRNA: ribosomal RNA; snRNA: small nuclear RNAs; Spodoptera frugiperda: S. frugiperda; B. mori: Bombyx mori; Cn(PPP3C): serine/threonine protein phosphatase 3, catalytic subunit; $5-\mathrm{HT}_{2} \mathrm{R}$ : serotonin receptor $2 \mathrm{C}$; NSCLC: non-small cell lung cancer; FBS: fetal bovine serum; RIP: RNA immunoprecipitation; FISH: fluorescent in situ hybridization; WST: 2-(2-methoxy-4-nitrophenyl)-3-(4-nitrophenyl)5-(2,4-disulfophenyl)-2H-tetrazolium, monosodium salt; MTT: 3-(4,5-dimethyl2-thiazolyl)-2,5-diphenyl-2-H-tetrazolium bromide; DAPI: 4',6-diamidino2-phenylindole; ASO: antisense oligo nucleotide; NR: non-redundant; COG: Cluster of Orthologous Group; GO: Gene Ontology; IAP: apoptosis inhibitor; Bcl2: B-cell lymphoma 2; Apaf1: apoptotic protease-activating factor 1; Gas5: growth arrest specific 5; TNF: tumor necrosis factor; TRAIL: tumor necrosis factor-related apoptosis inducing ligand.

\section{Authors' contributions}

DDL and YCK designed the experiments; BW, LH, WJQ and XL perform the experiments; YWS and YPL analyzed the transcriptome data; $Z L Y, X M L, B C$ and SDX analyzed the experimental results, HLQ, RQ and LGY help to write the manuscript. All authors read and approved the final manuscript.

\section{Acknowledgements}

We thank Dr. Jingchen Sun for providing the S. frugiperda Sf9 cell line.

\section{Competing interests}

The authors declare that they have no competing interests, including specific financial interests and relationships and affiliations relevant to the subject of this manuscript.

\section{Availability of data and materials}

The transcriptome data are available from the NCBI GEO with the number of GSE98196.

\section{Consent for publication}

Not applicable.

Ethics approval and consent to participate

Not applicable.

\section{Funding}

This work was supported by the National Natural Science Foundation of China (31100938 and 31470114), Young Elite Scientist Sponsorship Program by China Association for Science and Technology (YESS 20150026) and Henan Basic and Frontier Technology Research Program (162300410133). 


\section{Publisher's Note}

Springer Nature remains neutral with regard to jurisdictional claims in published maps and institutional affiliations.

Received: 19 October 2018 Accepted: 25 March 2019

Published online: 11 April 2019

\section{References}

1. Kiss T. Small nucleolar RNAs: an abundant group of noncoding RNAs with diverse cellular functions. Cell. 2002;109(2):145-8

2. Li D, Wang Y, Zhang K, Jiao Z, Zhu X, Skogerboe G, Guo X, Chinnusamy $\checkmark$, Bi L, Huang $Y$, et al. Experimental RNomics and genomic comparative analysis reveal a large group of species-specific small non-message RNAs in the silkworm Bombyx mori. Nucleic Acids Res. 2011;39(9):3792-805.

3. Kawaji H, Nakamura M, Takahashi Y, Sandelin A, Katayama S, Fukuda S, Daub CO, Kai C, Kawai J, Yasuda J, et al. Hidden layers of human small RNAs. BMC Genomics. 2008:9:157.

4. Ender C, Krek A, Friedlander MR, Beitzinger M, Weinmann L, Chen W, Pfeffer S, Rajewsky N, Meister G. A human snoRNA with microRNA-like functions. Mol Cell. 2008;32(4):519-28.

5. Saraiya AA, Wang CC. snoRNA, a novel precursor of microRNA in Giardia lamblia. PLoS Pathog. 2008;4(11):e1000224.

6. Brameier M, Herwig A, Reinhardt R, Walter L, Gruber J. Human box C/D snoRNAs with miRNA like functions: expanding the range of regulatory RNAs. Nucleic Acids Res. 2011;39(2):675-86

7. Scott MS, Avolio F, Ono M, Lamond Al, Barton GJ. Human miRNA precursors with box H/ACA snoRNA features. PLoS Comput Biol. 2009:5(9):e1000507.

8. Ono M, Scott MS, Yamada K, Avolio F, Barton GJ, Lamond Al. Identification of human miRNA precursors that resemble box C/D snoRNAs. Nucleic Acids Res. 2011;39(9):3879-91.

9. Yu F, Bracken CP, Pillman KA, Lawrence DM, Goodall GJ, Callen DF, Neilsen PM. p53 represses the oncogenic Sno-MiR-28 derived from a SnoRNA. PLoS ONE. 2015;10(6):e0129190.

10. Patterson DG, Roberts JT, King VM, Houserova D, Barnhill EC, Crucello A, Polska CJ, Brantley LW, Kaufman GC, Nguyen M, et al. Human snoRNA-93 is processed into a microRNA-like RNA that promotes breast cancer cell invasion. NPJ Breast Cancer. 2017;3:25.

11. Zhong F, Zhou N, Wu K, Guo Y, Tan W, Zhang H, Zhang X, Geng G, Pan $T$, Luo $H$, et al. A SnoRNA-derived piRNA interacts with human interleukin-4 pre-mRNA and induces its decay in nuclear exosomes. Nucleic Acids Res. 2015;43(21):10474-91.

12. He X, Chen $X$, Zhang X, Duan X, Pan T, Hu Q, Zhang Y, Zhong F, Liu J, Zhang $H$, et al. An Lnc RNA (GAS5)/SnoRNA-derived piRNA induces activation of TRAIL gene by site-specifically recruiting MLL/COMPASSlike complexes. Nucleic Acids Res. 2015:43(7):3712-25.

13. Stepanov GA, Filippova JA, Komissarov AB, Kuligina EV, Richter VA, Semenov DV. Regulatory role of small nucleolar RNAs in Human diseases. Biomed Res Int. 2015;2015:206849.

14. Jinn S, Brandis KA, Ren A, Chacko A, Dudley-Rucker N, Gale SE, Sidhu $R$, Fujiwara $H$, Jiang $H$, Olsen BN, et al. snoRNA U17 regulates cellular cholesterol trafficking. Cell Metab. 2015;21(6):855-67.

15. Zheng D, Zhang J, Ni J, Luo J, Wang J, Tang L, Zhang L, Wang L, Xu J, Su $B$, et al. Small nucleolar RNA 78 promotes the tumorigenesis in nonsmall cell lung cancer. J Exp Clin Cancer Res. 2015;34(1):49.

16. Dupuis-Sandoval F, Poirier M, Scott MS. The emerging landscape of small nucleolar RNAs in cell biology. Wiley Interdiscip Rev RNA. 2015;6(4):381-97.

17. Kishore S, Stamm S. The snoRNA HBII-52 regulates alternative splicing of the serotonin receptor 2C. Science. 2006;311(5758):230-2.

18. Runte M, Varon $R$, Horn D, Horsthemke B, Buiting K. Exclusion of the C/D box snoRNA gene cluster HBII-52 from a major role in Prader-Willi syndrome. Hum Genet. 2005;116(3):228-30.

19. Jha P, Agrawal R, Pathak P, Kumar A, Purkait S, Mallik S, Suri V, Chand Sharma M, Gupta D, Suri A, et al. Genome-wide small noncoding RNA profiling of pediatric high-grade gliomas reveals deregulation of several miRNAs, identifies downregulation of snoRNA cluster HBII-52 and delineates H3F3A and TP53 mutant-specific miRNAs and snoRNAs. Int J Cancer. 2015;137:2343-53.

20. Thorenoor N, Slaby O. Small nucleolar RNAs functioning and potential roles in cancer. Tumour Biol. 2015;36(1):41-53.

21. Williams GT, Farzaneh F. Are snoRNAs and snoRNA host genes new players in cancer? Nat Rev Cancer. 2012;12(2):84-8.

22. Gumienny R, Jedlinski DJ, Schmidt A, Gypas F, Martin G, Vina-Vilaseca A Zavolan M. High-throughput identification of C/D box snoRNA targets with CLIP and RiboMeth-seq. Nucleic Acids Res. 2017;45(5):2341-53.

23. Li R, Fox AH. SPArking interest in the long noncoding RNA world: a new class of 5' SnoRNA-stabilized LncRNA that influences alternative splicing. Mol Cell. 2016;64(3):435-7.

24. Falaleeva M, Pages A, Matuszek Z, Hidmi S, Agranat-Tamir L, Korotkov K, Nevo Y, Eyras E, Sperling R, Stamm S. Dual function of C/D box small nucleolar RNAs in rRNA modification and alternative pre-mRNA splicing. Proc Natl Acad Sci U S A. 2016;113(12):E1625-34.

25. Chen H, Qiang H, Fan K, Wang S, Zheng Z. The snoRNA MBII-52 regulates cocaine-induced conditioned place preference and locomotion in mice. PLOS ONE. 2014;9(6):e99986.

26. Soeno Y, Taya Y, Stasyk T, Huber LA, Aoba T, Huttenhofer A. Identification of novel ribonucleo-protein complexes from the brain-specific snoRNA MBII-52. RNA. 2010;16(7):1293-300.

27. Kishore S, Khanna A, Zhang Z, Hui J, Balwierz PJ, Stefan M, Beach C, Nicholls RD, Zavolan M, Stamm S. The snoRNA MBII-52 (SNORD 115) is processed into smaller RNAs and regulates alternative splicing. Hum Mol Genet. 2010;19(7):1153-64.

28. Doe CM, Relkovic D, Garfield AS, Dalley JW, Theobald DE, Humby T, Wilkinson LS, Isles AR. Loss of the imprinted snoRNA mbii-52 leads to increased 5htr2C pre-RNA editing and altered 5HT2CR-mediated behaviour. Hum Mol Genet. 2009;18(12):2140-8.

29. Mei YP, Liao JP, Shen J, Yu L, Liu BL, Liu L, Li RY, Ji L, Dorsey SG, Jiang ZR, et al. Small nucleolar RNA 42 acts as an oncogene in lung tumorigenesis. Oncogene. 2012;31(22):2794-804.

30. Chen WD, Zhu XF. Small nucleolar RNAs (snoRNAs) as potential non-invasive biomarkers for early cancer detection. Chin J Cancer. 2013;32(2):99-101.

31. Mannoor K, Liao J, Jiang F. Small nucleolar RNAs in cancer. Biochim Biophys Acta. 2012;1826(1):121-8.

32. Xu B, Ye MH, Lv SG, Wang QX, Wu MJ, Xiao B, Kang CS, Zhu XG. SNORD47, a box C/D snoRNA, suppresses tumorigenesis in glioblastoma. Oncotarget. 2017;8(27):43953-66.

33. Mourtada-Maarabouni M, Pickard MR, Hedge VL, Farzaneh F, Williams GT. GAS5, a non-protein-coding RNA, controls apoptosis and is downregulated in breast cancer. Oncogene. 2009;28(2):195-208.

34. Xu G, Yang F, Ding CL, Zhao LJ, Ren H, Zhao P, Wang W, Qi ZT. Small nucleolar RNA 113-1 suppresses tumorigenesis in hepatocellular carcinoma. Mol Cancer. 2014;13:216.

35. Ideue T, Hino K, Kitao S, Yokoi T, Hirose T. Efficient oligonucleotide-mediated degradation of nuclear noncoding RNAs in mammalian cultured cells. RNA. 2009;15(8):1578-87.

36. Liang XH, Vickers TA, Guo S, Crooke ST. Efficient and specific knockdown of small non-coding RNAs in mammalian cells and in mice. Nucleic Acids Res. 2011;39(3):e13.

37. Ploner A, Ploner C, Lukasser M, Niederegger H, Huttenhofer A. Methodological obstacles in knocking down small noncoding RNAs. RNA. 2009;15(10):1797-804.

38. Liang XH, Sun H, Nichols JG, Crooke ST. RNase H1-dependent antisense oligonucleotides are robustly active in directing RNA cleavage in both the cytoplasm and the nucleus. Mol Ther. 2017;25(9):2075-92.

39. Pickard MR, Williams GT. Regulation of apoptosis by long non-coding RNA GAS5 in breast cancer cells: implications for chemotherapy. Breast Cancer Res Treat. 2014;145(2):359-70.

40. Shi X, Sun M, Liu H, Yao Y, Kong R, Chen F, Song Y. A critical role for the long non-coding RNA GAS5 in proliferation and apoptosis in non-smallcell lung cancer. Mol Carcinog. 2015;54(Suppl 1):E1-12.

41. Tortoriello G, Accardo MC, Scialo F, Angrisani A, Turano M, Furia M. A novel Drosophila antisense scaRNA with a predicted guide function. Gene. 2009;436(1-2):56-65.

42. Klumpp S, Krieglstein J. Serine/threonine protein phosphatases in apoptosis. Curr Opin Pharmacol. 2002;2(4):458-62. 
43. Selth LA, Gilbert C, Svejstrup JQ. RNA immunoprecipitation to determine RNA-protein associations in vivo. Cold Spring Harb Protoc. 2009;2009(6):pdb prot5234.

44. Querido E, Dekakra-Bellili L, Chartrand P. RNA fluorescence in situ hybridization for high-content screening. Methods. 2017;126:149-55.

45. Marrosu E, Ala P, Muntoni F, Zhou H. Gapmer antisense oligonucleotides suppress the mutant allele of COL6A3 and restore functional protein in ullrich muscular dystrophy. Mol Ther Nucleic Acids. 2017;8:416-27.

46. Conesa A, Gotz S, Garcia-Gomez JM, Terol J, Talon M, Robles M. Blast2GO: a universal tool for annotation, visualization and analysis in functional genomics research. Bioinformatics. 2005;21(18):3674-6.

47. Ye J, Fang L, Zheng H, Zhang Y, Chen J, Zhang Z, Wang J, Li S, Li R, Bolund L. WEGO: a web tool for plotting GO annotations. Nucleic Acids Res. 2006;34(Web Server issue):W293-7.
48. Xia Q, Zhou Z, Lu C, Cheng D, Dai F, Li B, Zhao P, Zha X, Cheng T, Chai C, et al. A draft sequence for the genome of the domesticated silkworm (Bombyx mori). Science. 2004;306(5703):1937-40.

49. Nandakumar S, Ma H, Khan AS. Whole-genome sequence of the Spodoptera frugiperda Sf9 insect cell line. Genome Announc. 2017;5(34):e00829-17.

50. Negre V, Hotelier T, Volkoff AN, Gimenez S, Cousserans F, Mita K, Sabau X, Rocher J, Lopez-Ferber M, d'Alencon E, et al. SPODOBASE: an EST database for the lepidopteran crop pest Spodoptera. BMC Bioinformatics. 2006;7:322.

51. Zuker M. Mfold web server for nucleic acid folding and hybridization prediction. Nucleic Acids Res. 2003;31(13):3406-15.
Ready to submit your research? Choose BMC and benefit from:

- fast, convenient online submission

- thorough peer review by experienced researchers in your field

- rapid publication on acceptance

- support for research data, including large and complex data types

- gold Open Access which fosters wider collaboration and increased citations

- maximum visibility for your research: over $100 \mathrm{M}$ website views per year

At BMC, research is always in progress.

Learn more biomedcentral.com/submissions 